Original Paper http://ajol.info/index.php/ijbcs http://indexmedicus.afro.who.int

\title{
Influence des substrats fibre de coco, coque d'arachide et compost de soja sur le comportement de deux variétés de tomates TMA97 et Lindo en culture hydroponique
}

\author{
Noupé Diakaria COULIBALY ${ }^{1 *}$, Lassina FONDIO ${ }^{1}$, Mako François De Paul N'GBESSO ${ }^{1}$, \\ Yannick BRINDOU ${ }^{2}$, Christian Landry OSSEY $^{1}$ et André Gabazé GADJI ${ }^{1}$ \\ ${ }^{1}$ CNRA (Centre National de Recherche Agronomique), 01 BP 633 Bouaké 01, Côte d'Ivoire. \\ ${ }^{2}$ Université Nangui Abrogoua, 02 BP 801 Abidjan 02, Côte d'Ivoire. \\ *Auteur correspondent ; E-mail: noupediakaria@gmail.com
}

\begin{tabular}{ccc}
\hline Received: 20-09-2020 & Accepted: 22-02-2021 & Published: 28-02-2021 \\
\hline
\end{tabular}

\section{RESUME}

Pour réduire les difficultés des populations urbaines à l'accès à la terre et aussi pour améliorer la qualité de la production maraîchère en évitant les maladies telluriques, la mise au point d'un substrat adapté pour la culture hydroponique a été entreprise. Deux variétés de tomate, TMA97 et Lindo ont été évaluées sur trois substrats : fibres de coco, compost de soja et coques d'arachide. Les observations et mesures ont porté sur la croissance végétative, le délai de floraison puis les paramètres du rendement. Les résultats obtenus ont montré que la meilleure croissance a été obtenue avec la variété Lindo sur les fibres de coco. Le taux de fruits avarié le plus élevé (37\%) a également été produit par Lindo sur ce substrat. Par contre, la variété TMA97 cultivée sur le compost de soja en a donné le plus faible (1,3\%). Statistiquement, les rendements potentiels et nets ont été identiques chez les variétés de tomates étudiées. Mais numériquement, les rendements nets ont été plus élevés chez TMA97 que chez Lindo quel que soit le substrat utilisé. Chez la variété Lindo, les rendements nets les plus importants ont été obtenus avec le compost de soja. Les coques d'arachide ont donné les faibles rendements. Concernant la variété TMA97, les valeurs les plus élevées ont été produites sur les fibres de coco ; alors que les plus petites valeurs ont été observées sur les coques d'arachide.

(C) 2021 International Formulae Group. All rights reserved.

Mots clés : Tomate, hydroponie, solution nutritive, substrat, rendement.

\section{Influence of coconut fiber, peanut shell and soybean compost substrates on the behavior of two tomato varieties TMA97 and Lindo in hydroponic cultivation}

\begin{abstract}
To reduce the urban population's difficulties in accessing land and also to improve the quality of vegetable production by avoiding telluric diseases, the development of a suitable substrate for hydroponic cultivation has been undertaken. Two varieties of tomato, TMA97 and Lindo were evaluated on three substrates:
\end{abstract}


coconut fibers, soy compost and peanut shells. Observations and measurements focused on vegetative growth, flowering time, and yield parameters. The results obtained showed that the best growth was obtained with the Lindo variety on coconut fibres. The highest rate of damaged fruit (37\%) was also produced by Lindo on this substrate. On the other hand, the variety TMA97 grown on soy compost gave the lowest (1.3\%). Statistically, potential and net yields were identical in the tomato varieties studied. But numerically, the net yields are higher in TMA97 than in Lindo regardless of the substrate used. In the Lindo variety, the highest net yields were obtained with soy compost. Peanut shells gave low yields. For the TMA97 variety, the highest values were produced on coconut fibers; while the smallest values were observed on peanut shells.

Keywords : Tomato, hydroponics, nutrient solution, substrate, yield.

\section{INTRODUCTION}

La tomate (Solanum lycopersicum L) est une plante herbacée, très cultivée pour son fruit consommé à l'état frais ou transformée. Elle constitue le deuxième légume, le plus consommé dans le monde après la pomme de terre (INRA, 2010). Selon le FAO (2013), la Chine, les Etats-Unis et la Turquie en sont les plus grands producteurs avec respectivement 48,$58 ; 12,6$ et 11 millions de tonnes de tomate fraîche par an, contre 32364 tonnes pour la Côte d'Ivoire. Cette production demeure faible pour répondre aux besoins des populations ivoiriennes estimés à plus de 100000 tonnes par an (Soro et al., 2007). La faible production s'explique en autres, par les conditions climatiques défavorables, le non-respect des itinéraires techniques, les attaques de nombreux ravageurs et maladies (Bado, 2002 ; Samba et al., 2013). Aussi, la croissance démographique et l'urbanisation entraînent une augmentation de la demande en tomate. Il apparaît alors important de sélectionner des variétés et de mettre au point des techniques culturales mieux adaptées aux contraintes pour accroître la production en toute saison (Noupé et al., 2019). Généralement, l'agriculture urbaine et périurbaine participe de façon intégrée à la lutte contre la pauvreté et à l'assainissement environnemental (Parrot et al., 2008 ; Conchita et al., 2010). Elle participe à l'assainissement des villes en utilisant comme engrais, les déchets recyclés et les eaux usées traitées (Compaoré et al., 2010). Les producteurs urbains pratiquent le marâichage de façon continue sur les mêmes parcelles à cause de l'insuffisance de terres cultivables. C'est le cas des grandes agglomérations de Côte d'Ivoire, où les maraîchers cultivent généralement les terrains non encore bâtis et les bordures des cours d'eau (Affou et al., 2013). Pour accroître le rendement de leurs cultures, les producteurs sont obligés d'apporter de nombreux types d'engrais et autres pesticides à fortes doses et à des fréquences élevées (Michel et al., 2010). L'emploi excessif des fertilisants et pesticides dans les zones urbaines et périurbaines a été décrit comme une contrainte dangereuse au développement des productions légumières dans les pays africains (Diao, 2004 ; Perpétue et al., 2019).

C'est dans ce contexte que la culture hydroponique a été introduite en Côte d'Ivoire en 2008 pour répondre aux objectifs suivants : (i) faciliter l'accès des populations urbaines aux produits maraîchers de qualité, (ii) réduire les difficultés d'accès à la terre, (iii) contribuer à la création d'emplois pour les jeunes, les femmes et (iv) occuper les personnes âgées. Cependant, la diffusion de cette technologie a rencontré de nombreuses difficultés tant au niveau de la disponibilité du substrat fibres de coco et des divers engrais pour constituer les solutions nutritives. En outre, elle nécessite une main d'œuvre qualifiée pour produire la solution nutritive et pratiquer une gestion rationnelle de la culture hydroponique.

Pour un meilleur développement de la technique hydroponique dans le pays, le Centre National de Recherche Agronomique (CNRA) se propose de tester d'autres substrats, d'identifier les matériaux locaux permettant de 
réduire le coût de mise en place et de déterminer la rentabilité économique d'une culture hydroponique de production des légumes les plus demandés par les populations. La présente étude est initiée pour évaluer l'effet de trois substrats à base de sous-produits agricoles sur deux variétés de tomate en culture hydroponique au centre de la Côte d'Ivoire. L'objectif général de cette étude était de proposer un substrat peu onéreux et disponible dans l'environnement du producteur qui pourrait se substituer aux fibres de coco.

\section{MATERIEL ET METHODES Matériel végétal}

Cette étude a porté sur deux variétés de tomate; TMA97 et Lindo. La première est une variété sélectionnée par le CNRA, la seconde est une variété commercialisée. Le choix de la tomate se justifie du fait de sa plus forte consommation et des difficultés de la production de la tomate dans les conditions ordinaires du sol.

\section{Méthodes d'étude \\ Dispositif expérimental}

Les traitements observés au cours de cet essai ont été les suivants :

- 2 variétés de tomate (TMA97 et Lindo) ;

- 3 substrats de culture (compost à base de soja, coques d'arachide et fibres de coco).

Le dispositif expérimental adopté pour ce module hydroponique, a été des blocs aléatoires avec trois rangés de bacs (Figure 1). Chaque rangé est composé de huit bacs de 1,5 $\mathrm{m} \times 1 \mathrm{~m}$ chacun. A chaque rangé correspond un substrat de culture. Les plants de tomate ont été repiqués sur les parcelles élémentaires (bacs) de $1,5 \mathrm{~m}^{2}$. Dans chaque bac, les plants ont été repiqués sur deux (02) lignes de quatre (04) plants à raison de huit (08) plants par bac (Figure 2). La distance de plantation a été de 40 $\mathrm{cm}$ entre plants et $50 \mathrm{~cm}$ entre lignes. Une rangé de huit bacs comprend 48 plants de chaque variété. Chaque traitement a été répété quatre (04) fois. Ce dispositif a été installé sous un apatam dont le toit était couvert avec un plastique imperméable qui laisse passer la lumière (Figure 3).

\section{Préparation des substrats et mise en place des cultures \\ Préparation des substrats}

Dans cette étude, trois types de substrats à base de sous-produits agricoles locaux ont été utilisés. Les coques d'arachide et les fibres de coco alimentées par une solution nutritive. Le compost à base de résidus de récolte de soja a été arrosé à l'aide d'eau de robinet.

\section{- Substrat fibre de coco (FC)}

Ce substrat est la référence en culture hydroponique. La fibre a été obtenue auprès d'une société de production des fibres de coco installée dans la ville d'Assinie (Sud de la Côte d'Ivoire). Il a été plongé dans de l'eau pendant 10 jours puis pressées et séchées au soleil pendant 7 jours. Après séchage, le substrat a été mis dans les bacs de culture. Cette immersion permet d'éliminer les parasites contenus dans le substrat.

\section{* Substrat coques d'arachide (CA)}

Les coques d'arachide ont été collectées auprès des décortiqueuses d'arachide de la ville de Niakara (Centre de la Côte d'Ivoire) qui est une grande zone de production d'arachide. Les coques collectées ont été immergées dans des fûts d'eau pendant 10 jours. Ceci permet de réduire leur capacité de fermentation par élimination des éléments fermentescibles comme les sucres. En outre, cette immersion dans l'eau contribue à stériliser le substrat par l'élimination de certains pathogènes ne supportant pas cette asphyxie (bactéries, champignons, insectes...). Ensuite, les coques d'arachide ont été pressées puis séchées au soleil pendant 7 jours. Après séchage, le substrat ainsi obtenu a été mis dans les bacs de culture.

\section{* Substrat compost de Soja (CS)}

Le substrat compost de soja est le résultat du compostage de résidus de récolte de soja collectés sur des parcelles à la Station de Recherche sur les Cultures Vivrières de 
Bouaké (Centre de la Côte d'Ivoire). Le compostage consiste à faire fermenter, dans les conditions contrôlées, des déchets organiques en présence de l'oxygène de l'air. Le compostage s'est effectué à l'air libre pendant six (06) mois. Ce substrat tout comme les précédents a subi l'immersion pendant 10 jours puis pressées et séchées au soleil pendant 7 jours, neutralisant ainsi les parasites. Après séchage, il a été mis dans les bacs pour la culture.

\section{- Solution nutritive}

Les substrats à base de coque d'arachide et de la fibre de coco ont été alimentés par une solution nutritive mise au point par Fondio et al. (2013). Pour l'obtenir, il faut dissoudre dans 10001 d'eau, les quantités des engrais tels que Callifert (100 g), NPK00-23-19 (500 g), Super Latex (300 g), Nitrate de calcium (600 g) et NPK12-24-18 (700 g). La conductivité électrique de la solution est de l'ordre de 2,154 $\mathrm{dS} / \mathrm{m}$.

\section{Mise en place des cultures}

Pour la réalisation de la pépinière, les graines de tomate ont été semées dans des alvéoles en polyéthylène. Les alvéoles ont été préalablement remplies avec un substrat composé de compost traité la veille avec du furadan. Elles étaient arrosées tous les jours avec de l'eau. Ensuite, les alvéoles ont été conservées pendant trois (03) jours dans une salle à l'abri du soleil pour assurer les conditions favorables pour une germination optimale. En cas d'observation de symptômes d'attaque, les plantules atteintes sont éliminées des alvéoles.

Après une période de 27 jours en pépinière, les plants de tomate ont été repiqués dans les bacs remplis avec les différents substrats élaborés. Ils ont été repiqués à raison de 8 plants par bacs ; soit 2 lignes de 4 plants.

\section{* Méthode de fertigation}

Après le repiquage des plants dans les bacs, ils ont été arrosés avec de l'eau de robinet deux fois par jour pendant trois (03) jours afin qu'ils s'adaptent à leur milieu de culture. Après cette période d'acclimatation, des arrosages journaliers à raison de deux arrosages par jours (la matinée et la soirée) ont suivi jusqu'à la fin du cycle de culture. En effet, les bacs contenant les substrats inertes (coques d'arachide et fibres de coco) ont été arrosés avec de l'eau contenant la solution nutritive pendant 2 heures. Les bacs contenant le compost de soja ont été arrosés avec de l'eau de robinet pendant la même durée. Cet arrosage a constitué l'activité principale pour le suivi des plants. Des arrosages d'appoint ont été faits avec l'eau de robinet pendant les périodes caniculaires.

A chaque séance d'arrosage, les goutteurs sont vérifiés pour s'assurer que l'eau ou la solution nutritive coule normalement. Ensuite, la même solution nutritive est réutilisée à condition qu'elle ne soit pas trop acide. Pour ce faire, une prise du pH à l'aide du $\mathrm{pH}$-mètre se fait régulièrement.

\section{Observations et mesures expérimentales}

Les observations et les mesures ont porté sur la hauteur des plants, la vitesse de croissance des plants, nombre de bouquets floraux observés par semaine, le délai de 50\% floraison, le taux de nouaison des fruits (rapport du nombre moyen de fruits comptés un (01) mois après floraison sur le nombre de corymbes comptés) et les composantes du rendement (nombre de fruits, nombre de fruits avariés, poids des fruits récoltés, poids des fruits avariés, poids des fruits sains).

\section{Méthode d'analyse des données}

Les données obtenues ont été analysées à l'aide des logiciels Excel 2013 et STATISTICA version 7.1. Excel 2013 a servi à confectionner les matrices d'analyse et la construction des graphiques. Les analyses de variance et la comparaison des moyennes ont réalisées à l'aide de STATISTICA 7.1. Le test de Duncan a été choisi pour la comparaison des moyennes lorsque l'analyse des variances révèle des différences significatives entre les traitements au seuil de probabilité de 5\%. 
Fût solution nutritive

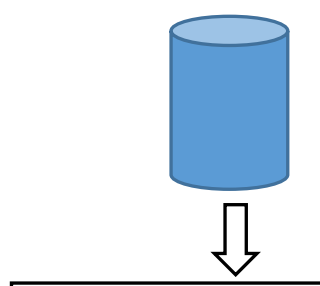

Coque d'arachide + TMA97

(CA V1R1)

Coque d'arachide + LINDO

(CA V2R1)

Coque d'arachide + TMA97

(CA V1R2)

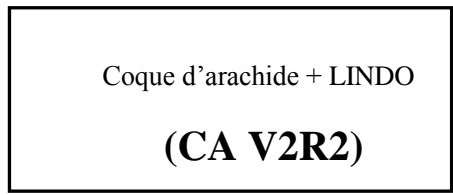

Coque d'arachide + TMA97

(CA V1R3)

Coque d'arachide + LINDO

(CA V2R3)

Coque d'arachide + TMA97

(CA V1R4)

Coque d'arachide + LINDO

(CA V2R4)
Fût solution

nutritive
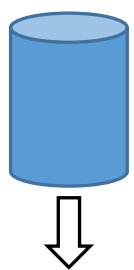

Fibre de coco + LINDO

(FC V2R1)

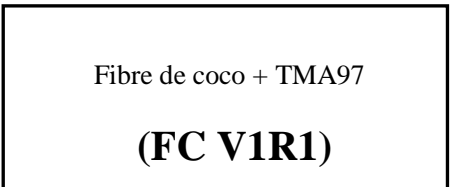

Fibre de coco + LINDO

(FC V2R2)

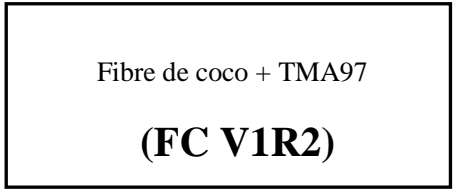

Fibre de coco + LINDO

(FC V2R3)

Fibre de coco + TMA97

(FCV1R3)

Fibre de coco + LINDO

(FC V2R4)

Fibre de coco + TMA97

(FC V1R4)
Fût eau
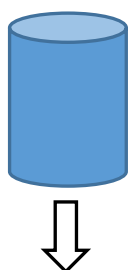

Compost Soja + TMA97

(CS V1R1)

Compost Soja + LINDO

(CS V2R1)

Compost Soja + TMA97

(CS V1R2)

Compost Soja + LINDO

(CS V2R2)

Compost Soja + TMA97

(CS V1R3)

Compost Soja + LINDO

(CS V2R3)

Compost Soja + TMA97

(CS V1R4)

Compost Soja + LINDO

(CS V2R4)

Figure 1 : Dispositif expérimental du module hydroponique. 


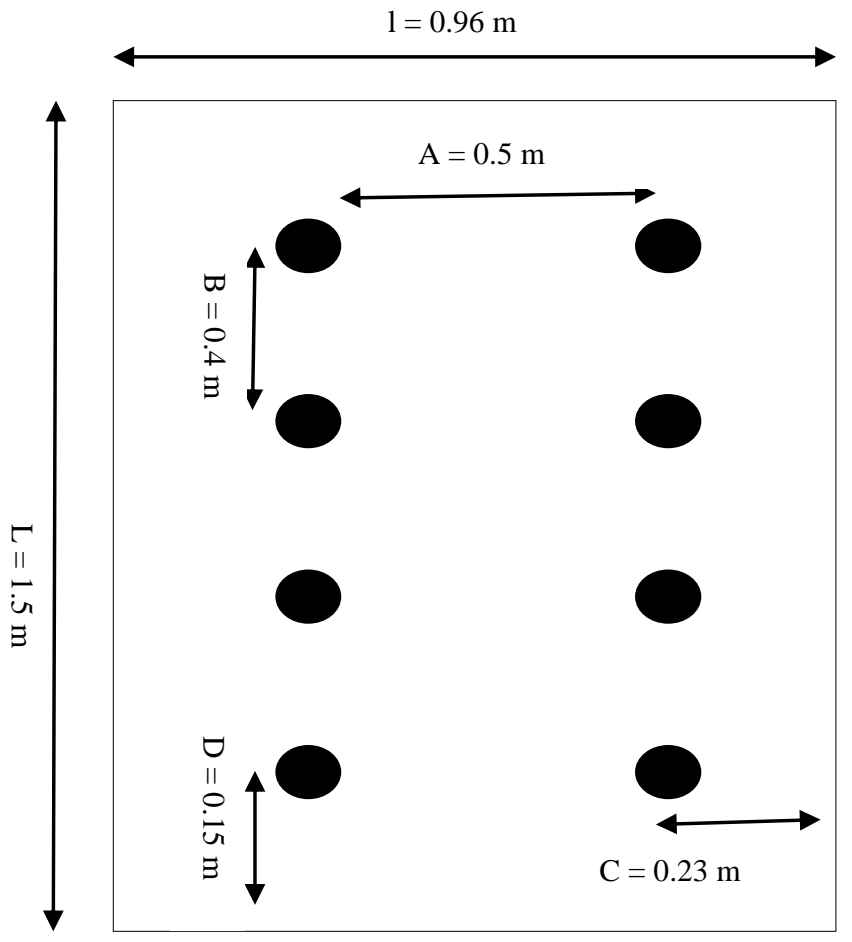

Figure 2 : détail d'une parcelle élémentaire (bac de culture).

L: Longueur du bac ; $\mathbf{l}$ : Largeur du bac ; $\mathbf{A}$ : Distance de repiquage entre lignes ; $\mathbf{B}$ : Distance entre deux plants ; $\mathbf{C}$ : Distance entre les plants et la bordure de la longueur du bac ; D : Distance entre les plants et la bordure de la largeur du bac.

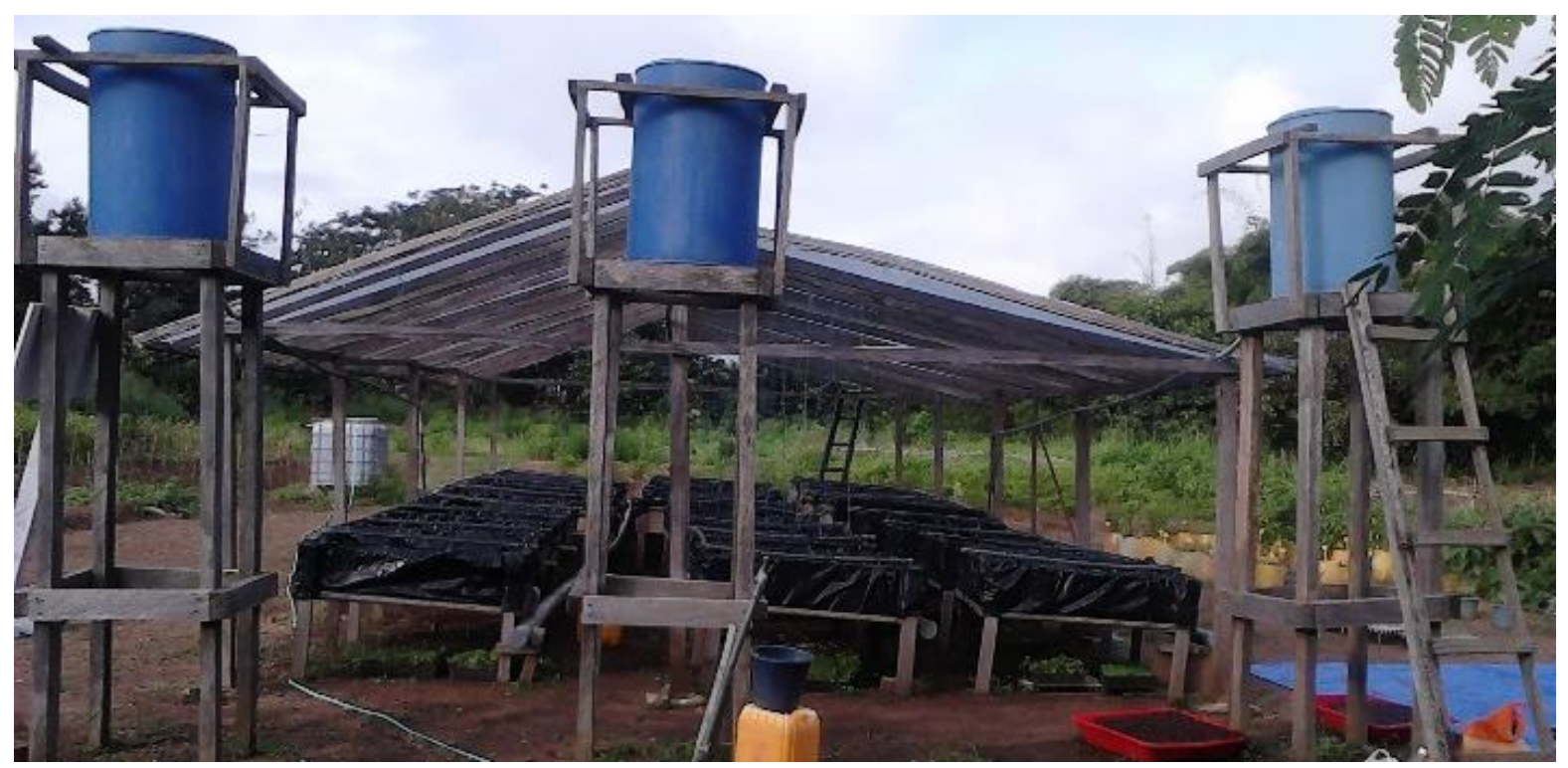

Figure 3 : Module hydroponique. 


\section{RESULTATS}

\section{Paramètres végétatifs \\ Hauteur moyenne des tiges}

L'analyse de la Figure 4 a montré qu'il y a une évolution progressive de la hauteur des tiges au cours du temps chez toutes les plantes. Les plus grandes hauteurs des tiges ont été obtenues avec les fibres de coco quelle que soit la variété utilisée. Le substrat à base de coques d'arachide a donné les plus faibles valeurs. Il a été noté que pour un même substrat, les valeurs de la hauteur de la tige sont plus importantes chez la variété Lindo par rapport à celle de TMA97.

\section{Vitesse de croissance des plants}

La Figure 5 illustre la vitesse de croissance des plants en fonction du substrat au cours du temps. Les résultats obtenus ont indiqué que la vitesse de croissance est plus importante dans le substrat fibres de coco que ceux du compost et des coques d'arachide. La vitesse de croissance a été plus élevée chez la variété Lindo que celle de TMA97 quel que soit substrat utilisé.

\section{Phénologie \\ Délai de floraison des plants et hauteur plants au stade floraison}

Le Tableau 1 regroupe le délai de floraison et la hauteur des plants au stade de la floraison. Les résultats de l'analyse de variance ont indiqué qu'il n'y a aucune différence significative entre les valeurs obtenues pour ces paramètres étudiés.

\section{Rendements et ses composantes Nombre et taux des fruits}

Dans le Tableau 2 sont consignés le nombre total de fruits, le nombre total de fruits sains, le nombre de fruits avariés, le taux de fruits saints et le taux des fruits avariés. L'analyse de ce tableau a montré qu'il n'existe aucune différence significative entre les substrats en rapport avec le nombre total de fruits et le nombre de fruits sains. Par contre, en ce qui concerne le nombre de fruits avariés, les taux de fruits sains et avariés, une variabilité a été notée entre les différents substrats utilisés.
Un effet variété a également été noté. La variété Lindo cultivée sur les coques d'arachide et TMA97 sur le compost de soja ont eu le nombre de fruits avariés le plus petit ; alors que Lindo sur les fibres de coco en a donné la valeur la plus grande. Pour le taux de fruits sains, la plus importante valeur a été produite par TMA97 sur les substrats compost de soja et fibres de coco. Lindo sur les fibres de coco a généré la plus faible valeur. L'analyse de variance des taux de fruits avariés a montré que proportionnellement aux taux de fruits saints, les plus petites valeurs ont été enregistrées chez TMA97 sur les substrats compost de soja et fibres de coco.

\section{Poids des fruits}

Le Tableau 3 regroupe le poids total de fruits, le poids de fruits sains et le poids de fruits avariés. D'après les résultats obtenus, relativement au poids total de fruits et au poids de fruits sains, il n'a existé aucune différence significative entre les substrats et ceci indépendamment de la variété étudiée. Cependant, en ce qui concerne le poids de fruits avariés, des différences significatives ont été observées selon le substrat et la variété. En effet, TMA97 sur le compost de soja a donné la valeur la plus faible et la plus importante valeur a été enregistrée chez Lindo sur les fibres de coco.

\section{Taux de nouaison et rendements}

Le Tableau 4 présente le taux de nouaison, le rendement potentiel et le rendement net. Les résultats consignés dans ce tableau ont indiqué que les valeurs sont statistiquement identiques pour tous les paramètres étudiés. Numériquement, les rendements nets ont été plus élevés chez TMA97 que chez Lindo quel que soit le substrat utilisé. Chez la variété Lindo, les rendements nets les plus importants ont été obtenus avec le compost de soja. Les coques d'arachide ont donné les faibles rendements. Concernant la variété TMA97, les valeurs les plus élevées ont été produites sur les fibres de coco ; alors que les valeurs les plus petites ont été observées sur les coques d'arachide. 


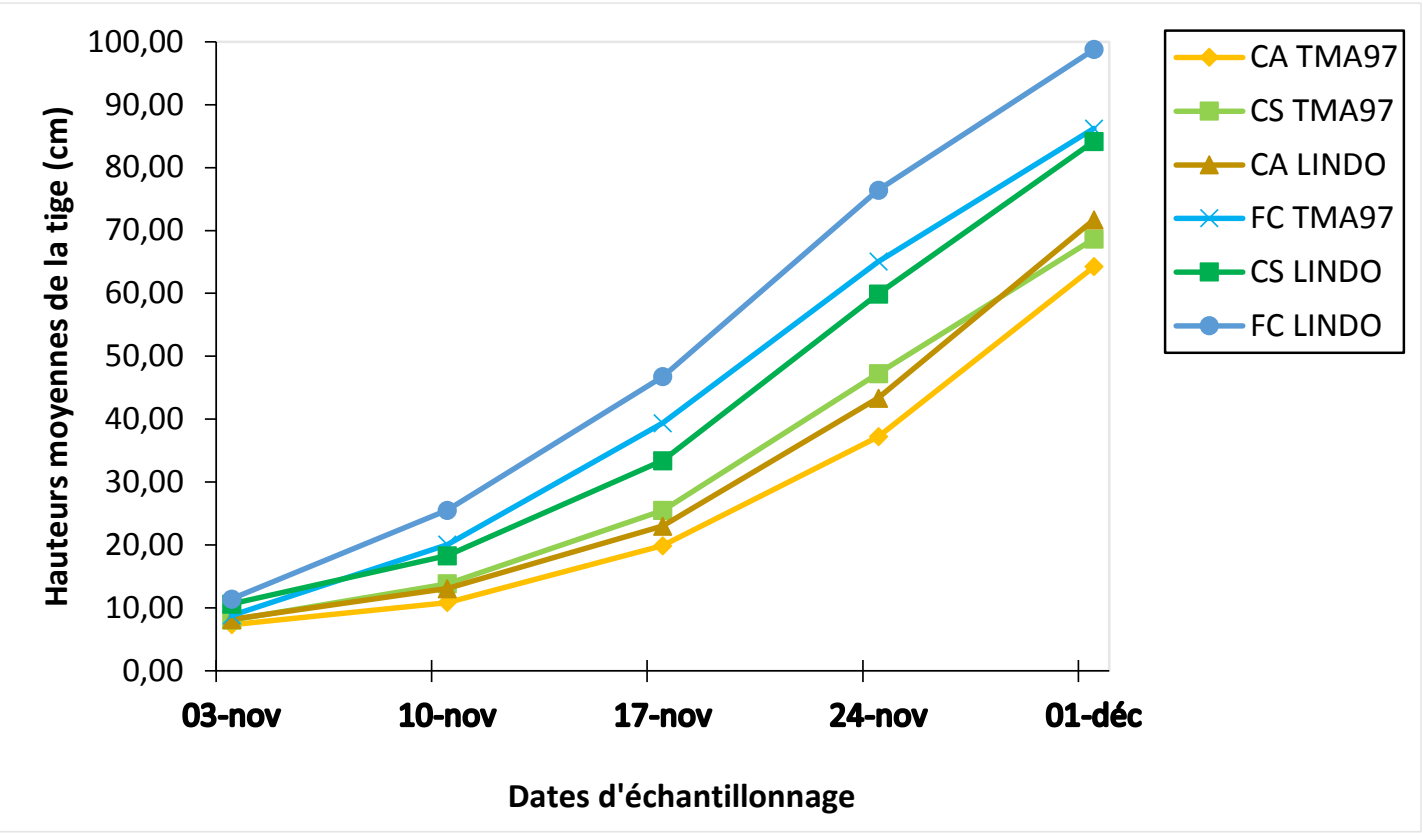

Figure 4 : Evolution de la hauteur moyenne des plants de tomate en fonction du temps et du substrat.

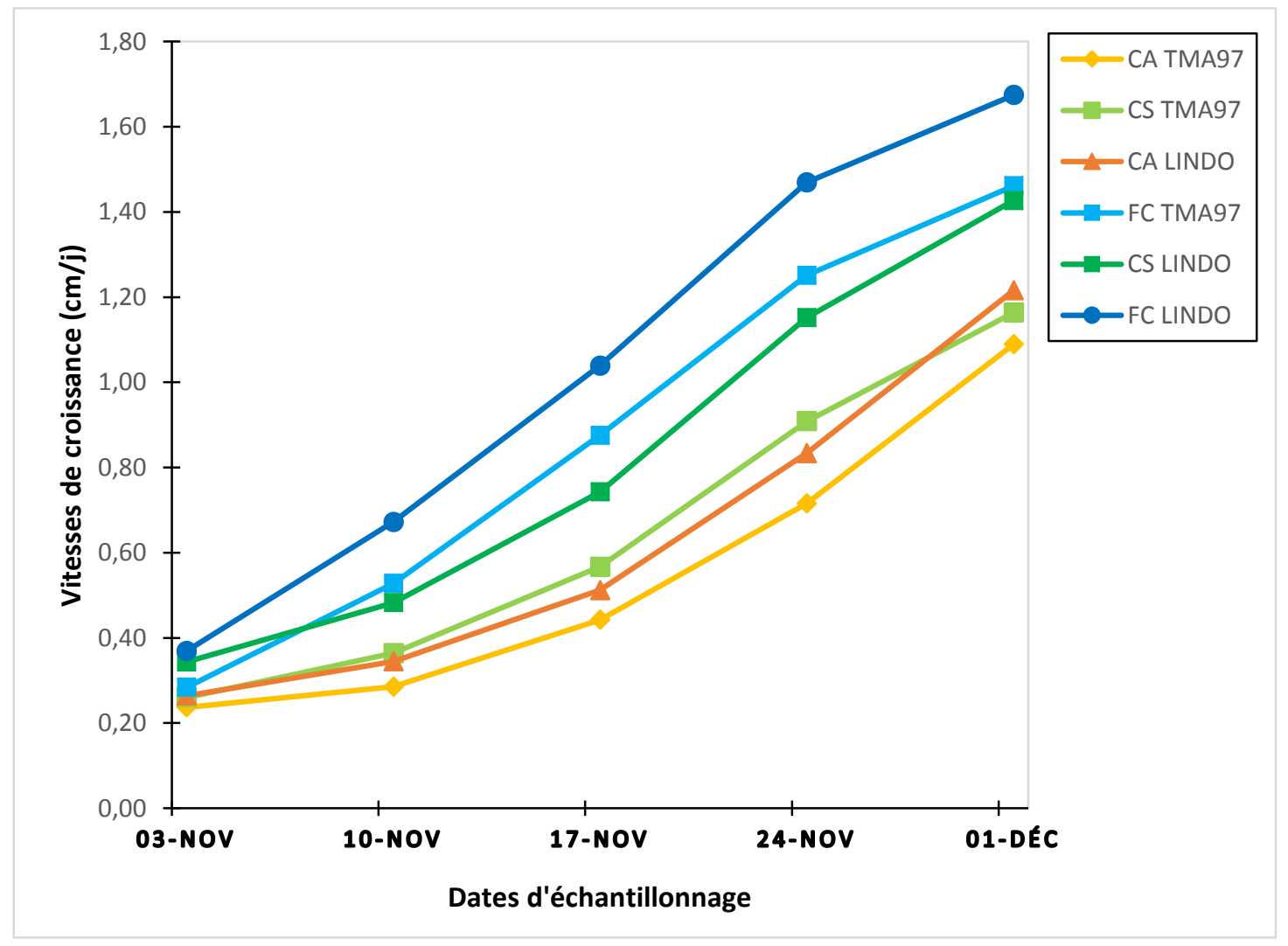

Figure 5 : Evolution de la vitesse de croissance des plants de tomate en fonction du temps et du substrat. 
Tableau 1 : Délai de floraison des plants et hauteur des plants au stade de floraison.

\begin{tabular}{lcc}
\hline Traitements & Hauteur plants stade floraison $(\mathbf{c m})$ & Délai de floraison (Jours) \\
\hline CA LINDO & $71,72 \mathrm{a}^{*}$ & $58 \mathrm{a}^{*}$ \\
CA TMA97 & $64,25 \mathrm{a}$ & $57 \mathrm{a}$ \\
CS LINDO & $59,91 \mathrm{a}$ & $54 \mathrm{a}$ \\
CS TMA97 & $52,31 \mathrm{a}$ & $55 \mathrm{a}$ \\
FC LINDO & $76,41 \mathrm{a}$ & $52 \mathrm{a}$ \\
FC TMA97 & $65,06 \mathrm{a}$ & $53 \mathrm{a}$ \\
\hline Moyenne & 64,94 & 55 \\
Probabilité & 0,81201 & 0,66690 \\
Significativité & NS & NS \\
CV $(\%)$ & 10,45 & 2,90 \\
\hline *Les chiffres en colonnes affectés de la même lettre ne diffèrent pas significativement au seuil de 5\% (Test de Duncan)
\end{tabular}

Tableau 2 : Nombre total de fruits, nombre total de fruits sain, nombre de fruits avariés, taux de fruits sain et taux des fruits avariés.

\begin{tabular}{lccccc}
\hline Traitements & $\begin{array}{c}\text { Nombre } \\
\text { total fruits }\end{array}$ & $\begin{array}{c}\text { Nombre fruits } \\
\text { sains }\end{array}$ & $\begin{array}{c}\text { Nombre fruits } \\
\text { avariés }\end{array}$ & $\begin{array}{c}\text { Taux fruits } \\
\text { sains }(\%)\end{array}$ & $\begin{array}{c}\text { Taux fruits } \\
\text { avariés }(\%)\end{array}$ \\
\hline CA LINDO & $116^{\mathbf{a}}$ & $75^{\mathbf{a}}$ & $4^{\mathbf{a}}$ & $76,78^{\mathbf{b}}$ & $23,22^{\mathbf{c}}$ \\
CA TMA97 & $135^{\mathbf{a}}$ & $118^{\mathbf{a}}$ & $17^{\mathbf{b}}$ & $87,97^{\mathbf{b c}}$ & $12,03^{\mathbf{b}}$ \\
CS LINDO & $144^{\mathbf{a}}$ & $107^{\mathbf{a}}$ & $37^{\mathbf{d}}$ & $86,51^{\mathbf{b c}}$ & $13,49^{\mathbf{b}}$ \\
CS TMA97 & $169^{\mathbf{a}}$ & $163^{\mathbf{a}}$ & $7^{\mathbf{a}}$ & $98,70^{\mathbf{c}}$ & $1,30^{\mathbf{a}}$ \\
FC LINDO & $262^{\mathbf{a}}$ & $113^{\mathbf{a}}$ & $149^{\mathbf{e}}$ & $63,01^{\mathbf{a}}$ & $36,99^{\mathbf{d}}$ \\
FC TMA97 & $256^{\mathbf{a}}$ & $223^{\mathbf{a}}$ & $27^{\mathbf{c}}$ & $94,40^{\mathbf{c}}$ & $5,60^{\mathbf{a}}$ \\
\hline Moyenne & 180 & 133 & 46 & 84,56 & 15,44 \\
Probabilité & 0,74105 & 0,36712 & 0,00002 & 0,0019 & 0,0019 \\
Significativité & $\mathrm{NS}$ & $\mathrm{NS}$ & $\mathrm{HS}$ & $\mathrm{HS}$ & $\mathrm{HS}$ \\
CV $(\%)$ & 23,29 & 29,55 & 36,85 & 6,32 & 34,63 \\
\hline
\end{tabular}

*Les chiffres en colonnes affectés de la même lettre ne diffèrent pas significativement au seuil de $5 \%$ (Test de Duncan)

Tableau 3 : Poids total de fruits, poids de fruits sains, poids de fruits avariés.

\begin{tabular}{lccc}
\hline Traitements & Poids total de fruits $(\mathbf{k g})$ & Poids de fruits sains $(\mathbf{k g})$ & Poids de fruits avariés $(\mathbf{k g})$ \\
\hline CA LINDO & $4,04^{\mathbf{a}}$ & $3,10^{\mathbf{a}}$ & $0,95^{\mathbf{b}}$ \\
CA TMA97 & $5,36^{\mathbf{a}}$ & $4,7^{\mathbf{a}}$ & $0,59^{\mathbf{b}}$ \\
CS LINDO & $4,95^{\mathbf{a}}$ & $4,36^{\mathbf{a}}$ & $0,59^{\mathbf{b}}$ \\
CS TMA97 & $5,06^{\mathbf{a}}$ & $4,98^{\mathbf{a}}$ & $0,07^{\mathbf{a}}$ \\
FC LINDO & $5,90^{\mathbf{a}}$ & $3,74^{\mathrm{a}}$ & $2,16^{\mathbf{c}}$ \\
FC TMA97 & $7,75^{\mathbf{a}}$ & $7,32^{\mathbf{a}}$ & $0,43^{\mathbf{b}}$ \\
\hline Moyenne & 5,51 & 4,71 & 0,80 \\
Probabilité & 0,36712 & 0,0774 & 0,00001 \\
Significativité & $\mathrm{NS}$ & $\mathrm{NS}$ & $\mathrm{HS}$ \\
CV $(\%)$ & 22,30 & 26,11 & 26,16 \\
\hline
\end{tabular}

*Les chiffres en colonnes affectés de la même lettre ne diffèrent pas significativement au seuil de 5 \% (Test de Duncan) 
Tableau 4 : Taux de nouaison, rendement potentiel et rendement net.

\begin{tabular}{cccc}
\hline Traitements & Taux de nouaison $(\%)$ & Rendement potentiel (T/ha) & Rendement net (T/ha) \\
\hline CA LINDO & $68,99^{\mathbf{a}^{*}}$ & $26,96^{\mathrm{a}}$ & $20,64^{\mathrm{a}}$ \\
CA TMA97 & $63,78^{\mathbf{a}}$ & $35,74^{\mathrm{a}}$ & $31,83^{\mathrm{a}}$ \\
CS LINDO & $65,08^{\mathbf{a}}$ & $33,03^{\mathrm{a}}$ & $29,08^{\mathbf{a}}$ \\
CS TMA97 & $67,61^{\mathrm{a}}$ & $33,71^{\mathrm{a}}$ & $33,22^{\mathrm{a}}$ \\
FC LINDO & $70,98^{\mathrm{a}}$ & $39,34^{\mathrm{a}}$ & $24,94^{\mathrm{a}}$ \\
FC TMA97 & $64,35^{\mathrm{a}}$ & $51,65^{\mathrm{a}}$ & $48,77^{\mathrm{a}}$ \\
\hline Moyenne & 66,80 & 36,74 & 31,41 \\
Probabilité & 0,37728 & 0,36712 & 0,07740 \\
Significativité & $\mathrm{NS}$ & $\mathrm{NS}$ & $\mathrm{NS}$ \\
CV $(\%)$ & 10,29 & 22,30 & 26,11 \\
\hline
\end{tabular}

*Les chiffres en colonnes affectés de la même lettre ne diffèrent pas significativement au seuil de 5\% (Test de Duncan)

\section{DISCUSSION}

Les plants cultivés sur le substrat fibres de coco ont eu une meilleure croissance en hauteur par rapport à ceux cultivés sur le compost de soja et les coques d'arachide. Le comportement des variétés de tomate par rapport au milieu de culture s'est manifestée lors des premières étapes de reprise par une croissance végétative distincte d'un substrat de culture à un autre. Cet effet peut être attribué à la nature du substrat et à certaines caractéristiques physiques et physicochimiques qui composent le substrat. La fibre de coco et le compost semblent mieux stimuler les processus du développement que les coques d'arachide qui ont exercé peu d'influence. Ces résultats concordent avec ceux de Boufares (2012) qui a noté que pendant les phases de développement, la vigueur de croissance n'est pas nécessairement un caractère variétal comme cela est couramment admis, mais peut résulter aussi de certaines conditions du milieu.

Les résultats obtenus, en ce qui concerne les délais de floraison, ont permis d'observer une absence de variabilité entre les variétés étudiées quel que soit le substrat utilisé. Il semble que le gène qui contrôle la floraison ne diffère pas selon la variété de tomate; de plus, ce gène ne semble pas être influencé par les facteurs extérieurs tels que le milieu de culture. Cependant, selon les travaux de Fondio et al. (2013), la culture hydroponique de la tomate semble induire une précocité de floraison (30 JAR) par rapport à la culture sur sol (40 JAR).

La variété Lindo a produit plus de fruits que TMA97. Il est donc probable que Lindo soit potentiellement plus productive que TMA97. Le taux de fruits noncommercialisables élevé enregistré chez Lindo signifierait qu'elle semble plus sensible aux facteurs biotiques et abiotiques que TMA97. Les variétés de tomate cultivées sur le compost de soja ont été moins affectées par les ravageurs et maladies. Ceci pourrait s'expliquer par le fait des microorganismes stimulateurs de croissance des plantes et des agents de lutte biologique actifs présents dans le compost. Ces microorganismes, ont semblet-il, contribué à la résistante des plantes aux agents pathogènes, notamment ceux qui affectent la croissance des plantes. Ceci confirme les résultats de Mohamed (2006) qui ont révélé que le compost peut améliorer l'aptitude des plantes à résister aux maladies causées par les agents pathogènes foliaires en induisant une résistance systémique.

La variété Lindo sur les fibres de coco a eu un taux de nouaison élevé mais un rendement net inférieur à celui de la variété TMA97 du fait des pourritures apicales. La variété TMA97 parait plus tolérante à certains facteurs biotiques et donc finalement plus productive que Lindo avec un nombre de fruits saints plus élevé. En effet, plus le nombre de 
fruits saints est élevé, plus le rendement net augmente. Statistiquement, les rendements potentiels et nets ont été identiques chez les variétés de tomates étudiées. Mais numériquement, les rendements nets sont plus élevés chez TMA97 que chez Lindo quel que soit le substrat utilisé. Chez la variété Lindo, les rendements nets les plus importants ont été obtenus avec le compost de soja. Les coques d'arachide ont donné les plus faibles rendements. Concernant la variété TMA97, les valeurs les plus élevées ont été produites sur les fibres de coco; alors que les valeurs les plus petites ont été observées sur les coques d'arachide. D'après ces résultats, les rendements chez les variétés de tomate ont été plus élevés sur les fibres de coco que sur les coques d'arachide. Et pourtant, ces substrats ont la même source de fertilisation qui est la solution nutritive. La différence entre ces deux substrats pourrait se situer dans leur capacité de rétention en eau si l'on se réfère aux travaux de Boufares (2012). Selon lui, avec les substrats à faible capacité de rétention en eau, les plantes produisent moins de biomasse. Cependant, ni le rendement ni la qualité des fruits ne sont affectés. Une autre étude menée par Abbasi et al., (2002) a montré que, en plus de la stimulation de la croissance des plants de tomate, le rendement net de la tomate est plus élevé en utilisant le compost à base de résidus de récolte.

\section{Conclusion}

Selon les résultats obtenus, la variété de tomate TMA97 semble la plus productive sur les substrats de culture. Le rendement et les composantes de cette variété ont donc été les plus élevés sur les substrats de culture. Elle semble la mieux indiquée pour la culture hydroponique de la tomate en Côte d'Ivoire. Les rendements potentiels et net obtenus avec les deux variétés testées ont été statistiquement identiques quel que soit le substrat de culture utilisé. Ainsi, les substrats compost de soja et coques d'arachide peuvent remplacer le substrat fibres de coco (substrat de référence). Le substrat compost de soja ne nécessite pas de solution nutritive, donc plus économique que les deux autres substrats. De plus, il est disponible dans plusieurs zones agroécologiques de la Côte d'ivoire. En conséquence, le compost de soja serait le substrat le plus approprié pour la culture hydroponique.

\section{CONFLIT D'INTERETS}

Les auteurs ne déclarent aucun conflit d'intérêts.

\section{CONTRIBUTIONS DES AUTEURS}

NDC était l'investigateur principal ; MFDP, CLO, AGG et LF ont contribué à l'amélioration du manuscrit ; YB a effectué les observations et les relevés.

\section{REMERCIEMENTS}

Toute notre reconnaissance au FAO pour avoir été l'initiateur principal. Notre gratitude à l'endroit des autorités du Centre National de Recherche Agronomique (CNRA); notamment celles de la Station de Recherche sur les Cultures Vivrières à Bouaké pour avoir mis à notre disposition le matériel et la main d'œuvre pour la mise en place de l'expérimentation.

\section{REFERENCES}

Abbasi PA, Al-Dahmani J, Sahin F, Hoitink HAJ, Miller SA. 2002. Effect of compost amendments on disease severity and yield of tomato in conventional and organic production systems. Plant Disease, 86: 156-161.

Affou SW, Sebastien KO, Emma FA, Kablan T, Rose KN. 2013. Perception des risques sanitaires dans le maraîchage à Abidjan, Côte d'Ivoire. International Journal of Biological and Chemical Sciences, 7(5): 1829-1837.

Bado BV. 2002. Rôle des légumineuses sur la fertilité des sols ferralitiques tropicaux des zones guinéennes et soudaniennes du Burkina Faso. Thèse de doctorat à l'université de Laval, Québec, 166 p.

Boufares K. 2012. Comportement de trois variétés de pommes de terre (Spunta, Désirée et Chubaek) entre deux milieux de culture substrat et hydroponique. Mémoire pour l'obtention du diplôme de 
Magister en Agronomie. Université Aboubekr Belkaïd - Tlemcen (Algérie). $108 \mathrm{p}$.

Compaoré E, Nanema LS, Bonkoungou S, Sedogo MP. 2010. Évaluation de la qualité de composts de déchets urbains solides de la ville de Bobo-Dioulasso, Burkina Faso pour une utilisation efficiente en agriculture. Journal of Applied Biosciences, 33: 2076-2083.

Conchita MG, Kêdowidé M, Sedogo P, Cissé G. 2010. Dynamique spatio temporelle de l'agriculture urbaine à Ouagadougou: Cas du Maraîchage comme une activité montante de stratégie de survie. VertigO, 10(2). DOI : 10.4000/vertigo.10312.

Diao MB. 2004. Situation et contraintes des systèmes urbains et périurbains de production horticole et animale dans la région de Dakar. Cahier Agriculture, 13(1) : 39-49.

Fondio L, Djidji AH, N'gbesso MFDP. 2013. Formulation de solutions nutritives pour la culture hydroponique des légumes en Côte d'Ivoire. Rapport technique, Protocole d'accord CNRA/FAO, $31 \mathrm{p}$.

INRA. 2010. Légumes. Ephytia.inra.fr/ tomate/importance économique. 18/05/18

Parrot L, Njoya A, Temple L, Assogba KF, Kahane R, Diao B, Havard M. 2008. Agricultures et développement urbain en Afrique subsaharienne. In Colloque Agricultures et Développement Urbain en Afrique de l'Ouest et du Centre, 2005-1030/2005-11-03, Yaoundé, Cameroun. Le Harmattan, 208 p.

Perpétue MN, Koffi NOA, Fattoh EE, Vincent K. 2019. Assessment of the impact of pesticide use in urban and periurban agriculture in Abidjan, Côte d'Ivoire. International Journal of Biological and
Chemical Sciences, 13(6): 2824-2837. DOI:

https://dx.doi.org/10.4314/ijbcs.v13i6.32

Michel N, François L, Zacharie G, Noufou O, Michel PS. 2010. Les pratiques culturales traditionnelles appauvrissent les sols en zone des savanes du Tchad. International Journal of Biological and Chemical Sciences, 4(4): 871-881.

Miller SA, Sahin F, Krause M, Al-Dahmani J, Stone A, Hoitink H. 1997. Control of bacterial leaf spot of radish incompostamended planting mixes. Phytopathology. Plant Disease, 86(2) : 156-161.

Mohamed L. 2006. Influence de la qualité des composts et de leurs extraits sur la protection des plantes contre les maladies fongiques. Thèse de Doctorat. Université de Neuchâtel (Tunisie). 161 p.

Noupé DC, Lassina F, Mako FDPN, Bakary D. 2019. Evaluation des performances agronomiques de quinze nouvelles lignées de tomate en station au centre de la Côte d'Ivoire. International Journal of Biological and Chemical Sciences, 13(3): 1565-1581.

DOI: https://dx.doi.org/10.4314/ijbcs.v13i3.29

Samba T, Minamba B, Birama SC, Adama C. 2013. Cultures dans les zones sahéliennes de l'Afrique de l'Ouest : une condition sine qua none pour l'augmentation de la productivité et de la durabilité des systèmes de culture à base de mil, $25 \mathrm{p}$.

Soro S, Doumbia M, Dao D, Tschannen A, Girardin O. 2007. Performance de six cultivars de tomate Lycopersicon esculentum Mill. Contre la jaunisse en cuillère des feuilles, le flétrissement bactérien et les nématodes à galles. Sciences et Nature, 4 : 123-130. 